\title{
Los textos escolares en Ciencias Sociales y Ciencias NATURALES Y SU RELACIÓN CON LOS PROCESOS DE aprendizaje. El caso de Colombia
}

(Social and natural science school texts and their relationship to learning processes. The Colombian case)

Gillian Moss

(Universidad del Norte, Barranquilla, Colombia)

\section{Introducción}

Con respecto a las dos primeras preguntas que nos planteamos en la introducción general a este trabajo, a saber: “Cómo incide el lenguaje de los textos escolares en los procesos de aprendizaje?” y “¿Cómo interactúan maestr@syalumn@s con el texto?”, el Colectivo Urdimbre ${ }^{4}$ del Grupo Lenguaje y Educación de la Universidad del Norte en Barranquilla, Colombia, ha realizado varias investigaciones acerca de la relación entre el discurso de los textos escolares, la interacción texto-maestr@-estudiante que se genera en el aula alrededor del texto, los procesos de aprendizaje y los procesos de formación ciudadana. En el presente texto, haré referencia a los tres primeros aspectos.

\section{Rasgos discursivos de los textos escolares que causan dificultades de comprensión}

Con respecto al discurso de los textos escolares, en el transcurso de nuestras investigaciones, hemos analizado 9 textos, 4 de Ciencias Naturales y 5 de Ciencias Sociales 5 . Los análisis aplicados se basan principalmente en

\footnotetext{
${ }^{4}$ El Colectivo Urdimbre está conformado por Diana Ávila, Norma Barletta, Diana Chamorro, Jorge Mizuno, Gillian Moss y Carlina Tapia.

5 Estos textos, en Colombia, son elaborados y vendidos por editoriales privadas pero deben adecuarse a los lineamientos curriculares y estándares de competencias básicas para el área definidos por el Ministerio de Educación Nacional.
} 
la lingüística sistémico-funcional de Halliday (1985/1994/2004): transitividad y ergatividad, Tema, desarrollo temático, modalidad, metáfora léxica y gramatical, densidad léxica, valoración, unidades textuales y tipo de tópico (Davies, 1995). Al complementar este análisis lingüístico con el estudio etnográfico de aula, nos ha sido posible identificar ciertos rasgos discursivos de los textos que tienden a causar dificultades para los y las estudiantes:

- insuficiencia de unidades textuales (introducción, transición, conclusión)

- información incompleta

- desarrollo temático complejo

- nominalización

- densidad léxica

- ausencia de modalización

- uso excesivo de la negación

- términos difíciles no definidos

Estos resultados (Moss et al., 1998/2003) coinciden en gran medida con los resultados de análisis de textos escolares que se han llevado a cabo en otras partes del mundo (ver, por ejemplo, Davies y Greene, 1984; Halliday y Martin, 1993; Vallejos Llobet et al, 2004; entre otros). Sin embargo, a pesar de esta acumulación de estudios realizados a través del tiempo, no se ha percibido esfuerzos por parte de los autores y editores de textos escolares para mejorar la estructuración y redacción de sus textos. Por consiguiente, es necesario ir un paso más allá en el estudio, observar y analizar los procesos de aprendizaje y proponer formas de mediación de los textos que puedan implementar los y las maestros/as para lograr mejores niveles de aprendizaje en sus aulas.

\section{Los niveles de aprendizaje}

Compartimos la visión sociocultural del aprendizaje, derivada de las teorías de Vygotsky (Vygotsky, 1934/1986; Carretero, 1994; De Vecchi y Giordan, 2006), que concibe el conocimiento como un producto del 
intercambio social. Por consiguiente, nos interesa observar el aprendizaje en el contexto de la interacción en el aula, en el proceso de construcción del conocimiento y no solamente como un producto que se consigna en exámenes o pruebas. En este sentido, nuestros análisis de observaciones de clases, entrevistas con estudiantes y producción escrita de estudiantes nos han permitido identificar seis niveles de aprendizaje en el aula (Ávila y Tapia, 2004; Colectivo Urdimbre, 2000; Barletta y Moss, 1999). Los niveles son:

0. Enunciado no pertinente: la participación del/de la estudiante no tiene relación con el tema de la interacción.

1. Recitado mecánico: el/la estudiante reproduce textualmente las palabras de su docente o del texto.

2. Elaboración incorrecta: el/la estudiante dice algo que se considera "incorrecto" pero que demuestra interacción con las ideas expresadas en el texto o por el/la maestro/a. Esto lo consideramos como un nivel superior al nivel de recitado mecánico por lo que demuestra un intento de procesar ideas en vez de simplemente reproducir palabras.

3. Comprensión académica: el/la estudiante muestra comprensión literal de las ideas expresadas en el texto o por el/la docente pero no las relaciona con la vida cotidiana.

4. Interacción entre significados académicos y experienciales: el/la estudiante establece relaciones entre el conocimiento nuevo y su conocimiento experiencial de la vida cotidiana.

5. Transformación de significados de la experiencia: el/la estudiante alcanza a utilizar los nuevos conocimientos para transformar su forma de interpretar el mundo. Estos dos últimos niveles equivalen ya al aprendizaje significativo.

Nuestras observaciones nos han mostrado, además, que el paso por estos niveles de aprendizaje no es un proceso lineal, sino que tiene un movimiento pendular, mostrando avances y retrocesos a medida que se desarrolla la interacción de diferentes maneras. Este movimiento pendular se representa gráficamente en la Figura 1. 


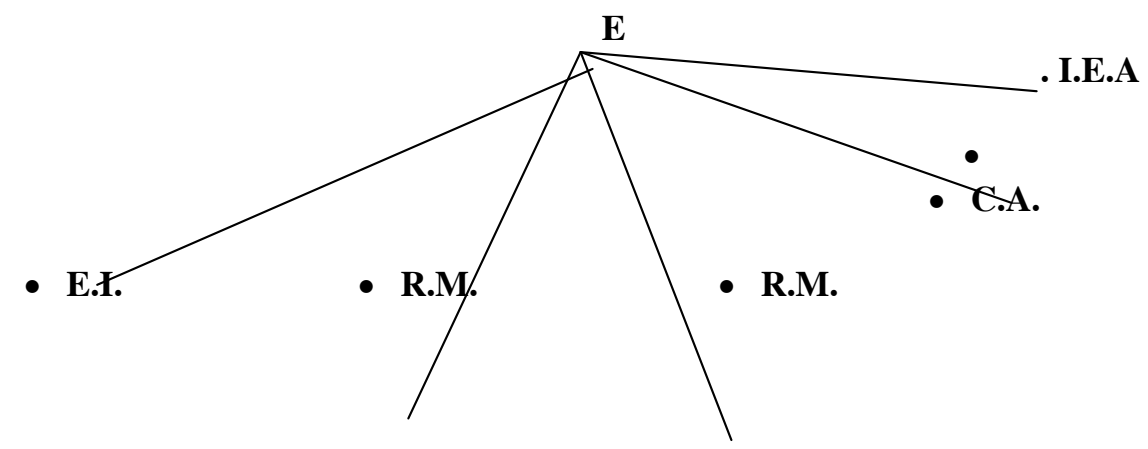

$\mathrm{E}=$ Estudiante

C.A. $=$ Comprensión académica

R.M = Recitado mecánico

E.I $=$ Elaboración incorrecta

I.A.E. $=$ Interacción entre significados académicos y experienciales

Figura 1

En este sentido, hemos podido observar que algunos tipos de interacción favorecen el proceso más que otros. Por ejemplo, los pocos ejemplos que hemos observado del nivel 5, ocurren cuando el/la estudiante se siente libre de evaluación y, por tanto, en libertad de correr riesgos y plantear interrogantes. Es por este motivo, entre otros, que consideramos absolutamente necesario observar el desarrollo del aprendizaje en su contexto de interacción en el aula.

\section{La mediación en el aula}

Sobra decir que en todo proceso de aula juega un papel fundamental la mediación del/de la docente. En una investigación acción basada en los resultados arriba mencionados, trabajamos con una maestra de Ciencias Sociales y una de Ciencias Naturales (Barletta y Mizuno, 2005; Ávila, 2001; Mizuno, 2001; Moss, 2001; Tapia, 2001). La metodología que seguimos contempló los siguientes pasos:

1. Análisis inicial, por parte del grupo investigador, de la unidad del texto a trabajar. 
2. Reunión con la profesora para ayudarla a reconocer probables fuentes de dificultad en el texto.

3. Observación de clase.

4. Reunión con la profesora para analizar el desarrollo de la clase.

5. Repetición de los dos últimos pasos hasta terminar la unidad.

Cada una de las dos profesoras desarrolló su propio estilo de mediación, en ambos casos con resultados alentadores en el sentido del logro de una mejoría en los niveles de aprendizaje evidenciados. Presento a continuación un resumen de las estrategias utilizadas por las dos maestras.

\subsection{Estrategias de la profesora de Ciencias Sociales}

- Desempaca las metáforas gramaticales.

- Trabaja con la terminología difícil.

- Completa la información, especialmente con respecto a las relaciones lógicas como causa-efecto.

- Provee unidades textuales pertinentes (introducción, transición, conclusión).

- Da voz al texto

La última estrategia, descrita también por Beck, McKeown and Worthy (1995), consiste en darle al texto algo de la naturaleza dialógica del discurso oral, identificar participantes humanos e imaginar sus reacciones ante las situaciones descritas.

\subsection{Estrategias de la profesora de Ciencias Naturales}

- Lectura de un fragmento de texto

- Clarificación de dudas acerca del texto

- Clarificación y explicación de conceptos

- Co-construcción con estudiantes de un mapa conceptual con términos claves del texto

- Oportunidades para que los y las estudiantes inicien la interacción 
Nuevamente, merece mención particular la última estrategia de la lista. Esta profesora, en varias ocasiones, propiciaba un ambiente muy amigable y de colaboración en el aula, en el que los y las estudiantes se sentían libres de evaluación y tomaban la iniciativa para hacer preguntas. En este contexto de responsabilidad compartida, fue que pudimos observar algunos cuantos casos del nivel 5 de aprendizaje: transformación de los significados de la experiencia.

\section{Conclusiones}

Queda evidente que gran número de textos escolares, en Colombia así como en otras partes del mundo, muestran una serie de rasgos discursivos que pueden dificultar su comprensión por parte de los y las estudiantes y, por ende, llevan a niveles de aprendizaje inferiores y poco significativos. Un trabajo colaborativo entre lingüistas y maestros/as puede facilitar la creación y aplicación de formas de mediar el texto en el aula que, en cierta medida, suplan las deficiencias del texto y permitan alcanzar niveles superiores de aprendizaje.

E-mail: mgilmoss@yahoo.com.br

\section{REFERENCIAS}

ÁviLA, D. 2001. Características Discursivas de los textos de Ciencias Sociales y su manejo en el aula. Memorias I Coloquio Internacional y III Regional de la Cátedra UNESCO para la Lectura y la Escritura en América Latina para Aprender a pensar. Cali: Unesco - Universidad del Valle (Disponible en CD).

Avila, D. y TAPIA, C. 2004. Las preguntas de los estudiantes: Una manera de construir aprendizaje. Zona Próxima, (5), 74 - 85

Barletta, N. y Mizuno, J. 2005. Una propuesta para el manejo del lenguaje del texto de Ciencias Naturales. Zona Próxima (6), p. 32-47

Barletta, N. y Moss, G. 1999. Las palabras de los estudiantes como evidencia de aprendizaje II Encuentro Iberoamericano de Colectivos Escolares, 1999, México D.F..Lenguaje e integraciones: Memorias del II Encuentro Iberoamericano de Colectivos Escolares. Ciudad de México: Universidad Pedagógica Nacional, $96-103$.

Beck, I. L., McKeown, M. G. y Worthy, J. 1995. Giving a text voice can improve students understanding. Reading Research Quarterly, 50 (2), pp. 220-238. 
Carretero, M. 1994. Constructivismo y Educación. Zaragoza. Edelviver.

Colectivo Urdimbre 2000. Libros de texto y aprendizaje en la escuela. Sevilla: Díada.

Davies, F. 1995. Introducing Reading. Harmondsworth: Penguin.

Davies, F. y Greene, T. 1984. Reading for learning in the sciences, Edinburgh: Oliver and Boyd.

De Vecchi, G \& Giordan, A. 2006. Guía práctica para la enseñanza científica. Sevilla, Díada Editora.

HALLIDAY, M.A.K. 1985/94/2004. Introducing Functional Grammar London: Arnold.

Halliday, M.A.K. y Martin, J.R. 1993. Writing Science. Literacy and discursive power London: Falmer Press.

Mizuno, J. 2001. Una metodología para la lectura e interpretación de la información cotextual en un texto de Ciencias Naturales. Memorias I Coloquio Internacional y III Regional de la Cátedra UNESCO para la Lectura y la Escritura en América Latina para Aprender a pensar. Cali: Unesco Universidad del Valle (Disponible en CD).

Mizuno, J. y Moss, G. 2006. La ideología en la interacción maestro-textoestudiante en las clases de Ciencias Sociales y su repercusión en la educación ciudadana. Akademos 8, (2), 63-87.

Moss, G. 2001. Características de los textos de Ciencias Naturales: los Tipos de Tópico y Unidades Textuales. Memorias I Coloquio Internacional y III Regional de la Cátedra UNESCO para la Lectura y la Escritura en América Latina para Aprender a pensar. Cali: Unesco - Universidad del Valle (Disponible en CD).

Moss, G., Mizuno, J., Ávila, D., Barletta, N., Carreño, S., Chamorro, D y TAPIA, C. 1998/2003. Urdimbre del texto escolar. ¿Por qué resultan difíciles algunos textos? Barranquilla: Ediciones Uninorte.

Natale, L. y Stagnaro, D., "La reconstrucción del Golpe Militar de 1976 en manuales escolares argentinos". Ponencia presentada en el 4to Congreso Alsfal. Florianópolis,UFSC. Octubre de 2008.

TAPIA, C. 2001. Las Palabras de los Estudiantes: Evidencia de Aprendizaje. Memorias I Coloquio Internacional y III Regional de la Cátedra UNESCO para la Lectura y la Escritura en América Latina para Aprender a pensar. Cali: Unesco - Universidad del Valle (Disponible en CD).

Vallejos Llobet, P. (comp.), Del Río, N., García, M. González, N., Monti, C. y Palmucci, D. 2004. El discurso científico pedagógico. Aspectos de la textualización del "saber enseñado". Bahía Blanca (Arg.): Universidad Nacional del Sur.

Vygotsky, L. S. 1986. Thought and language. Cambridge, Massachusetts: MIT Press. (Original en ruso: 1934). 